\section{Soluble CD163: A Novel Biomarker with Diagnostic and Therapeutic Implications in Autoimmune Diseases}

\section{To the Editor:}

Macrophage activation and function are significantly linked with the pathogenesis of a variety of autoimmune diseases, such as rheumatic diseases including rheumatoid arthritis (RA) and systemic lupus erythematosus (SLE). However, the precise mechanisms involved in this correlation are still elusive. Noticeably, we recently read with great interest the article by Peng, et al $^{1}$ showing serum soluble CD163 (sCD163) levels, mainly secreted by monocyte/macrophage lineage, were significantly increased in the patients with polymyositis (PM) and dermatomyositis (DM) compared with healthy controls. Particularly, they highlighted the involvement of CD163+ macrophage infiltration in the muscle in the development of PM/DM. It is commonly suggested that $\mathrm{PM} / \mathrm{DM}$ is correlated with the autoimmune disorders, and moreover elevated sCD163 expression and CD163+ macrophage (M2 macrophage) activation are also closely associated with some other autoimmune diseases.

CD163 is a member of the scavenger receptors in the membrane, which are exclusively expressed and can be shed from monocytes and macrophages to induce sCD163 in response to variously pathological conditions. Functionally, patients with early RA had significantly higher plasma levels of sCD163 than after 9 months of treatment; these levels are associated with disease activity and predict radiographic progression ${ }^{2}$. Conversely, plasma sCD163 levels decreased significantly after 3 months of treatment from the OPERA (optimized treatment algorithm in early rheumatoid arthritis) trial $^{3}$, which correlated with the investigated disease activity markers. More importantly, the soluble form of CD163, sCD163, could be observed not only in plasma, but also in other body fluids such as cerebrospinal fluid (CSF) and synovial fluid (SF). In keeping with the local production of sCD163 in SF, increased proportion of CD163+ macrophages and decreased CD69+ lymphocyte numbers were observed in spondyloarthropathy (SpA) synovium, which reflected the dual involvement of CD163+ macrophages in both SpA synovitis with global inflammation and impairment of $\mathrm{T}$ cell activation ${ }^{4}$.

CD163+ macrophages are involved in the inflammation of the skin in patients with SLE, in whom the number of CD163+ macrophages and the concentration of serum sCD163 are significantly increased in comparison with healthy control subjects ${ }^{5}$. The levels of plasma sCD163 are upregulated in the patients with multiple sclerosis (MS) compared with healthy controls ${ }^{6}$, a factor associated with decreased plasma proinflammatory cytokine levels such as interleukin (IL)-12 and IL-6. More recently, Stilund, et al showed that a high sCD163 CSF/serum ratio in patients with MS may reflect macrophage activation in MS lesions ${ }^{7}$. Further, sCD163 is identified as a marker of disease activity in adult-onset Still's disease, which is found to be correlated with ferritin expression in both the lymph node and tonsil ${ }^{8}$.

Taken together, these results elucidated that sCD163 levels are useful predictors in the development of diverse autoimmune diseases. Thus, the pharmacological and genetic intervention of sCD163 might provide a novel strategy in the diagnosis and treatment of autoimmune diseases.

DEXI ZHOU, MD, Laboratory of Clinical Pharmacy, Wannan Medical College, and the Department of Pharmacy, Yijishan Hospital, Wannan Medical College, Wuhu, China; YAQIN WANG, MSci, Laboratory of
Clinical Pharmacy, Wannan Medical College, and the Department of Pharmacy, Yijishan Hospital, Wannan Medical College, Wuhu, China; LU CHEN, MSci, Laboratory of Clinical Pharmacy, Wannan Medical College, and the Department of Pharmacy, Yijishan Hospital, Wannan Medical College, Wuhu, China; WEN ZHANG, MSci, Laboratory of Clinical Pharmacy, Wannan Medical College, and the Department of Pharmacy, Yijishan Hospital, Wannan Medical College, Wuhu, China; JIAJIE LUAN, Associate Professor, Laboratory of Clinical Pharmacy, Wannan Medical College, and the Department of Pharmacy, Yijishan Hospital, Wannan Medical College, Wuhu, China. Supported by the Natural Science Foundation of Anhui province (Grant number: KJ2014A262) and the Foundation for Young Talents in College of Anhui province (Grant number: 2010SQRL175). Address correspondence to Associate Prof. Jiajie Luan, Department of Pharmacy in Yijishan Hospital of Wannan Medical College, Wuhu, Anhui Province, China 241001. E-mail: luanjiajie757@163.com

\section{REFERENCES}

1. Peng QL, Zhang YL, Shu XM, Yang HB, Zhang L, Chen F, et al. Elevated serum levels of soluble CD163 in polymyositis and dermatomyositis: associated with macrophage infiltration in muscle tissue. J Rheumatol 2015;42:979-87.

2. Greisen SR, Møller HJ, Stengaard-Pedersen K, Hetland ML, Horslev-Petersen K, Jorgensen A, et al. Soluble macrophage-derived CD163 is a marker of disease activity and progression in early rheumatoid arthritis. Clin Exp Rheumatol 2011;29:689-92.

3. Greisen SR, Møller HJ, Stengaard-Pedersen K, Hetland ML, Hørslev-Petersen K, Junker P, et al. Macrophage activity assessed by soluble CD163 in early rheumatoid arthritis: association with disease activity but different response patterns to synthetic and biologic DMARDs. Clin Exp Rheumatol 2015;33:498-502.

4. Baeten D, Møller HJ, Delanghe J, Veys EM, Moestrup SK, De Keyser F. Association of CD163+ macrophages and local production of soluble CD163 with decreased lymphocyte activation in spondylarthropathy synovitis. Arthritis Rheum 2004;50:1611-23.

5. Nakayama W, Jinnin M, Makino K, Kajihara I, Makino T, Fukushima S, et al. CD163 expression is increased in the involved skin and sera of patients with systemic lupus erythematosus. Eur J Dermatol 2012;22:512-7.

6. Fabriek BO, Møller HJ, Vloet RP, van Winsen LM, Hanemaaijer R, Teunissen CE, et al. Proteolytic shedding of the macrophage scavenger receptor CD163 in multiple sclerosis. J Neuroimmunol 2007; 187:179-86.

7. Stilund M, Reuschlein AK, Christensen T, Møller HJ, Rasmussen PV, Petersen T. Soluble CD163 as a marker of macrophage activity in newly diagnosed patients with multiple sclerosis. PloS One 2014;9:e98588.

8. Colafrancesco S, Priori R, Alessandri C, Astorri E, Perricone C, Blank M, et al. sCD163 in AOSD: a biomarker for macrophage activation related to hyperferritinemia. Immunol Res 2014; 60:177-83

J Rheumatol 2016;43:4; doi:10.3899/jrheum.151317 